\title{
(6) OPEN ACCESS \\ Determining the force required in arthroscopic evaluation to assess the stability of syndesmotic ankle injury: a cadaveric study
}

\author{
Pieter D'Hooghe, ${ }_{1}^{1}$ Monique C Chambers, ${ }^{2}$ MaCalus V Hogan, ${ }^{2}$ Volker Musahl, ${ }^{2}$ \\ Khalid Alkhelaifi, ${ }^{1}$ Tabben Montassar, ${ }^{3}$ Freddie H Fu, ${ }^{2}$ Jean-Francois Kaux ${ }^{4}$
}

${ }^{1}$ Department of Orthopaedic Surgery, Aspetar Orthopaedic and Sports Medicine Hospital, Doha, Qatar

${ }^{2}$ Department of Orthopaedic Surgery and Bioengineering, University of Pittsburgh School of Medicine, Pittsburgh,

Pennsylvania, USA ${ }^{3}$ Department of Sport Science, Aspetar Orthopaedic and Sports Medicine Hospital, Doha, Qatar ${ }^{4}$ Physical and Rehabilitation Medicine (SportS), University Hospital of Liège, Liège, Belgium

\section{Correspondence to}

Dr Pieter D'Hooghe, Department of Orthopaedic Surgery, Aspetar Orthopaedic and Sportsmedicine Hospital, Doha 29222, Qatar; pieter.orthopedie@gmail.com

Accepted 4 January 2019 Published Online First 3 February 2019

Check for updates

(C) International Society of Arthroscopy, Knee Surgery and Orthopaedic Sports Medicine 2019. Re-use permitted under CC BY-NC. No commercial reuse. Published by BMJ.

To cite: D'Hooghe $P$,

Chambers MC,

Hogan MCV, et al. JISAKOS

2019;4:100-104.

\section{ABSTRACT}

Introduction The diagnosis of isolated distal

tibiofibular syndesmotic ankle instability proves to be a challenge. Although diagnostic imaging has added value, it is limited in the detection of distal syndesmotic ankle instability. The gold standard remains intraoperative testing through arthroscopic probing while externally stressing the ankle in a sagittal direction. However, no validated arthroscopic guidelines have been established to distinguish a stable from an unstable syndesmotic ankle joint. This cadaveric study presents anatomical and biomechanical data that can help surgeons correctly identify isolated distal syndesmotic ankle instability. Objective The purpose of this study is to quantify the necessary forces applied during ankle arthroscopy to evaluate syndesmotic instability in freshly frozen cadaveric ankles.

Methods A total of 16 fresh frozen cadaveric (age 58-74 years) ankles were included in the study. A dynamometer was used to measure the force necessary for the shaver tip to be inserted into the distal tibiofibular joint with the ankle in a neutral position. Measurements were performed first with the syndesmosis intact, and again following progressive transection of the syndesmotic ligaments, along with distal fixation. Results Significant differences were noted in the mean force required between the anterior inferior tibiofibular ligament (AITFL)+interosseous ligament (IOL) and no ligament cut methods $(p<0.001$ between the AITFL+IOL and AITFL cut $(p<0.001 ; 95 \% \mathrm{Cl}$ 44.80 to 50.70$)$, and between the AITFL+IOL and AITFL+IOL+PITFL cut $(p<0.001)$. There were also significant differences in the necessary mean forces applied between the one-SB and two-SB methods $(p<0.001)$, between the one-SB and one-screw methods $(p=0.010)$, between the one-SB and two-screw methods $(p=0.01)$, between the two-SB and two-screw methods $(p=0.003)$ and between the one-screw and two-screw methods $(p<0.001)$. Significant differences were found between the AITFL+IOL cut and the one-SB $(p<0.001)$, the two-SB $(p<0.001)$, the one-screw $(p<0.001)$ and the two-screw $(p<0.001)$ methods.

Conclusions This cadaveric study provides biomechanical data that can assist the surgeon in the arthroscopic evaluation of syndesmotic injuries. The data from this study need to be clinically correlated to ultimately assist in improving the outcome of patients with syndesmotic ankle injuries. Our study offers to bridge the gap to the development of arthroscopic tools that can identify the need for surgical fixation to the syndesmosis based on the laxity of specific ankle ligaments that contribute to subtle instability.

\section{What are the new findings}

- This cadaveric study provides biomechanical data that can assist surgeons in the arthroscopic evaluation of syndesmotic injuries.

- This cadaveric study aims at bridging the gap to the development of arthroscopic tools that can identify the need for surgical fixation to the syndesmosis based on the laxity of specific ankle ligaments that contribute to subtle instability.

- Since there are no validated arthroscopic measurements available yet, that the arthroscopic surgeon can use to identify subtle distal syndesmotic ankle instability, this cadaveric study offers new data in the process.

Level of evidence Level V cadaveric study.

\section{INTRODUCTION}

The distal ankle syndesmosis is a fibrous articulation in which the opposing joint surfaces are joined by a complex of three ligaments. The anterior and posterior tibiofibular ligaments form the syndesmosis, along with the interosseous ligament. The inferior transverse tibiofibular ligament can be considered as a fourth syndesmotic ligament, but is rather an extension of the posterior tibiofibular ligament. ${ }^{12}$ In the absence of a fracture, an isolated syndesmotic injury can occur as a result of an external rotation force acting on the foot, leading to eversion of the talus within the ankle mortise and increased dorsiflexion or plantar flexion. ${ }^{3}$ It can also occur after traumatic supination in association with injury to the lateral ligaments. ${ }^{5}$ Such syndesmotic injuries have been increasingly recognised in athletes since they can be associated with long-term ankle dysfunction and loss of time from play. ${ }^{6-8}$

According to the West Point Ankle Grading System, three grades of syndesmotic injuries can be distinguished. ${ }^{1}$ A grade I injury involves a partial tear to the anterior inferior tibiofibular ligament (AITFL) with a normal ankle radiograph. Grade II indicates a complete rupture of the AITFL and a partial tear of the interosseous ligament (IOL) with a normal ankle radiograph, but a positive external rotation or squeeze test. There is no consensus regarding the stability of this injury pattern and they are often referred to as having latent instability. ${ }^{8}$ 


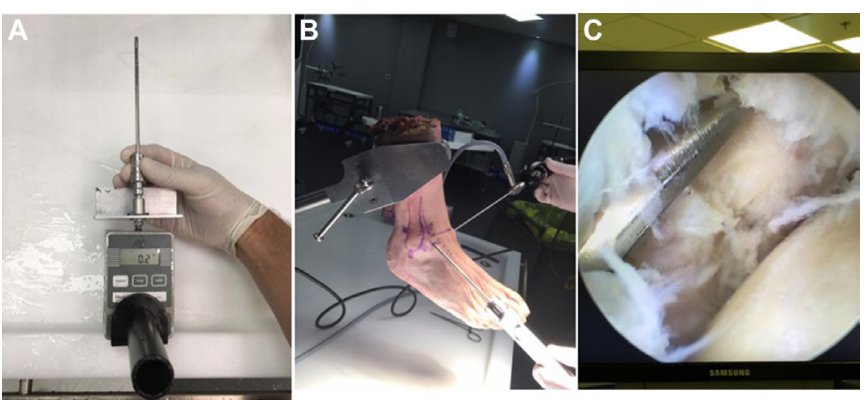

Figure 1 Arthroscopy set-up: (A) Presentation of the dynamometer and how the shaver is mounted onto it. The dynamometer shaver tip is inserted into the distal tibiofibular joint with the ankle in a neutral position during arthroscopy. (B) Overall set-up of the cadaveric specimen, portals, arthroscope and shaver during the testing procedures. (C) Intra-articular view of the anterolateral tibiotalar joint area with the shaver positioned during entry to the distal ankle syndesmosis.

Grade III injuries involve a complete disruption of the syndesmotic ligaments and a weight bearing ankle radiograph that is unstable with mortise widening. ${ }^{9}$

Stress radiographs and MRI can be helpful in the diagnosis of these injuries, but currently there is no best evidence-based test available that can identify syndesmotic instability, especially in grade II lesions. This is particularly relevant in the athletic population, where appropriate management is crucial for the player to return to the team. ${ }^{8}$ There is a consensus to use arthroscopy in the evaluation of syndesmotic stability in doubtful cases, but there is no surgical protocol available (except expert opinion) to identify syndesmotic stability under direct visualisation with arthroscopy. $^{9}$

The purpose of this study is to quantify the necessary forces applied during ankle arthroscopy to evaluate syndesmotic instability by using freshly frozen cadaveric ankles.

\section{MATERIALS AND METHODS}

A total of 16 freshly frozen cadaveric (age range, 58-74 years) ankles were used for this study. Foot and ankle specimens were secured using a clamp and the standard arthroscopic portals were established (figure 1). An intra-articular view was established to visualise the anterolateral tibiotalar joint as the shaver was inserted into the distal ankle syndesmosis (figure 1). A dynamometer (Aspetar Model 12-0343, 2017) was adjusted to a $4 \mathrm{~mm}$ arthroscopic shaver to measure the force necessary to enter a $4 \mathrm{~mm}$ shaver tip $1 \mathrm{~cm}$ above the tibiocrural joint line in the distal syndesmosis with the ankle in a neutral position during arthroscopy (figure 1). The dynamometer was trial tested

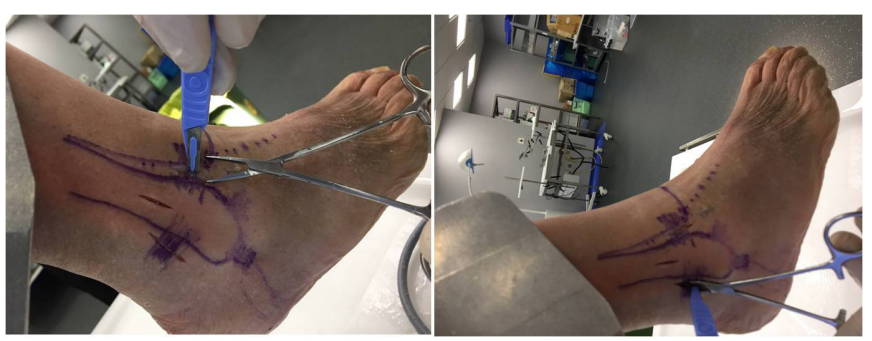

Figure 2 Ligament transections: Presentation on how the anterior inferior tibiofibular ligament and posterior inferior tbiofibular ligament are cut prior to testing.

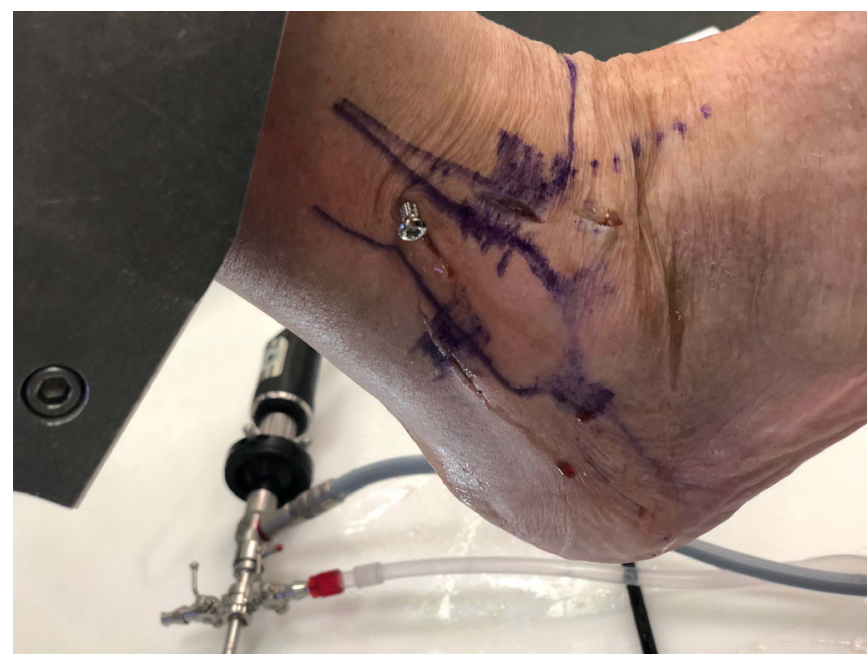

Figure 3 Screw fixation image showing how the screw was positioned (2-cortical, between $2 \mathrm{~cm}$ and $4 \mathrm{~cm}$ proximal from the tibiotalar joint and aimed at $30^{\circ}$ of anterior angulation).

to calibrate and assure measured accuracy based on 0.1 Newton. The measurement was performed first with the syndesmosis intact, then with subsequent cutting of the AITFL, the IOL and the posterior inferior tbiofibular ligament (PITFL)(figure 2). The force required to enter the $4 \mathrm{~mm}$ shaver tip, $1 \mathrm{~cm}$ proximal to the distal tibiotalofibular joint was measured.

After sectioning the AITFL and the IOL, two areas were prepared for syndesmotic suture button repair and two areas for syndesmotic screw fixation. A suture button was used to stabilise the syndesmosis and the shaver tip test was repeated. A second suture button was then introduced and the model was tested again. Next, the suture buttons were removed and one syndesmotic screw was introduced and the shaver test was repeated again. Finally, two screws were fixated for another shaver test check (figure 3). The suture button/screw fixation was only performed for the AITFL/IOL rupture combination, since the principal focus of this study is on the intraoperative decision making on grade II syndesmotic injuries. All of the surgical procedures were completed by the same trained orthopaedic surgeon.

\section{Statistical analysis}

Descriptive data are presented as means \pm SD. Kolmogorov's test was applied to test the normal distribution of the data. Levene's test was applied to control for parametrical assumptions for homogeneity of variance. The sphericity was tested by the Mauchly test. When the assumption of sphericity was not met, the significance of F-ratios was adjusted according to the Greenhouse-Geisser procedure. The forces applied for the four surgical methods (no ligament cut, AITFL cut, AITFL+IOLcut and AITFL+IOL+ PITFL cut) were compared through a one-way analysis of variance (ANOVA) for repeated measures. The same statistical analysis was used in order to compare the one-SB, the two-SB, the one-screw and the two-screw methods. Post hoc analysis included pairwise comparisons using Bonferroni interval adjustment to identify the significant differences. A paired-samples t-test was used to compare the AITFL+IOL cut method with the one-SB, the two-SB, the one-screw and the two-screw methods, respectively. The magnitude of the differences was assessed by effect sizes $(\eta 2) .{ }^{10}$ This analysis considers $\eta 2$ values as: small $(\eta 2=0.02)$, medium effect size $(\eta 2=0.13)$ 
Original article

\begin{tabular}{cccll}
\hline \multicolumn{4}{l}{ Table 1 } & \multicolumn{4}{l}{ Force required based on ligament deficiency } \\
\hline $\begin{array}{c}\text { Specimen } \\
\text { number }\end{array}$ & All intact & $\begin{array}{l}\text { AlTFL } \\
\text { deficient }\end{array}$ & $\begin{array}{l}\text { AlTFL+LOL } \\
\text { deficient }\end{array}$ & $\begin{array}{l}\text { Complete } \\
\text { transection }\end{array}$ \\
\hline 1 & $126 \mathrm{~N}$ & $102 \mathrm{~N}$ & $61 \mathrm{~N}$ & $58 \mathrm{~N}$ \\
2 & $132 \mathrm{~N}$ & $109 \mathrm{~N}$ & $66 \mathrm{~N}$ & $63 \mathrm{~N}$ \\
\hline 3 & $139 \mathrm{~N}$ & $110 \mathrm{~N}$ & $67 \mathrm{~N}$ & $62 \mathrm{~N}$ \\
4 & $119 \mathrm{~N}$ & $101 \mathrm{~N}$ & $56 \mathrm{~N}$ & $55 \mathrm{~N}$ \\
5 & $111 \mathrm{~N}$ & $98 \mathrm{~N}$ & $48 \mathrm{~N}$ & $45 \mathrm{~N}$ \\
6 & $136 \mathrm{~N}$ & $110 \mathrm{~N}$ & $68 \mathrm{~N}$ & $62 \mathrm{~N}$ \\
7 & $131 \mathrm{~N}$ & $108 \mathrm{~N}$ & $58 \mathrm{~N}$ & $58 \mathrm{~N}$ \\
8 & $121 \mathrm{~N}$ & $99 \mathrm{~N}$ & $49 \mathrm{~N}$ & $45 \mathrm{~N}$ \\
9 & $129 \mathrm{~N}$ & $104 \mathrm{~N}$ & $53 \mathrm{~N}$ & $51 \mathrm{~N}$ \\
10 & $137 \mathrm{~N}$ & $112 \mathrm{~N}$ & $63 \mathrm{~N}$ & $59 \mathrm{~N}$ \\
11 & $117 \mathrm{~N}$ & $99 \mathrm{~N}$ & $49 \mathrm{~N}$ & $46 \mathrm{~N}$ \\
12 & $130 \mathrm{~N}$ & $105 \mathrm{~N}$ & $56 \mathrm{~N}$ & $51 \mathrm{~N}$ \\
13 & $119 \mathrm{~N}$ & $102 \mathrm{~N}$ & $50 \mathrm{~N}$ & $45 \mathrm{~N}$ \\
14 & $141 \mathrm{~N}$ & $111 \mathrm{~N}$ & $65 \mathrm{~N}$ & $57 \mathrm{~N}$ \\
15 & $123 \mathrm{~N}$ & $106 \mathrm{~N}$ & $52 \mathrm{~N}$ & $52 \mathrm{~N}$ \\
16 & $129 \mathrm{~N}$ & $109 \mathrm{~N}$ & $60 \mathrm{~N}$ & $59 \mathrm{~N}$ \\
\hline
\end{tabular}

Individual force needed to enter the $4 \mathrm{~mm}$ shaver blade into the distal syndesmosis when: no ligament was cut, the AITFL was cut, the AITFL and IOL were cut, the AITFL, IOL and PITFL were cut (all three cut).

AITFL, anterior inferior tibiofibular ligament; IOL, interosseous ligament.

or large effect size $(\eta 2=0.26) .{ }^{10}$ Statistical significance was set at $\mathrm{p}<0.05$ and all analyses were carried out using SPSS V.20.0 programme for OS X (SPSS, Chicago, Illinois, USA).

\section{RESULTS}

\section{Comparison of ligaments transected}

The necessary mean forces, applied during the surgical procedure using the intact ligament, the AITFL cut, the AITFL +IOL cut and the AITFL+IOL+ PITFL cut methods are presented in table 1 .

ANOVA revealed that there was a main effect on the necessary force applied based on the method used for ligamentous transection $(\mathrm{F} 2.09,31.30=2458.13, \quad \mathrm{p}<0.001$, $\eta 2=0.994)$. Significant differences were found between the AITFL + IOL and no ligament cut methods $(\mathrm{p}<0.001 ; 95 \%$ CI 66.71 to 73.16$)$, between the AITFL+IOL and AITFL cut methods ( $\mathrm{p}<0.001 ; 95 \% \mathrm{CI} 44.80$ to 50.70 ), and between the AITFL+IOL and AITFL+IOL+ PITFL cut methods $(\mathrm{p}<0.001$; $95 \%$ CI 1.63 to 4.99 ). The force needed decreased by $25 \%$ when the AITFL was severed and by more half when the IOL was additionally severed. Additional section of the PITFL had a negligible effect.

\section{Comparison of fixation methods}

The necessary mean forces applied during the surgical procedure using the one-SB, the two-SB, the one-screw and the two-screw methods are detailed in table 2 and averages are presented in figure 4.

ANOVA showed a main effect of fixation type ( $F 3,45=40.21$, $\mathrm{p}<0.001, \eta 2=0.728)$ on the force applied throughout the surgical procedures using the SB and screw methods. Considering pairwise comparisons, significant differences were found between the one-SB and two-SB methods ( $\mathrm{p}<0.001 ; 95 \% \mathrm{CI}$ 2.9 to 7.46), between the one-SB and one-screw methods $(\mathrm{p}=0.010 ; 95 \% \mathrm{CI} 0.63$ to 5.61$)$, between the one-SB and two-screw methods ( $p=0.010 ; 95 \%$ CI 6.58 to 12.67$)$, between the two-SB and two-screw methods $(\mathrm{p}=0.003 ; 95 \%$ CI 1.39 to 7.49), between the one-screw and two-screw methods ( $\mathrm{p}<0.001 ; 95 \%$ CI 3.84 to 9.16). However, no significant

Table 2 Force required based on fixation type

\begin{tabular}{lllll}
\hline $\begin{array}{l}\text { Specimen } \\
\text { number }\end{array}$ & 1 SB & 2 SB & 1 screw & 2 screws \\
\hline 1 & $111 \mathrm{~N}$ & $114 \mathrm{~N}$ & $115 \mathrm{~N}$ & $117 \mathrm{~N}$ \\
2 & $119 \mathrm{~N}$ & $119 \mathrm{~N}$ & $124 \mathrm{~N}$ & $128 \mathrm{~N}$ \\
3 & $121 \mathrm{~N}$ & $125 \mathrm{~N}$ & $122 \mathrm{~N}$ & $125 \mathrm{~N}$ \\
4 & $109 \mathrm{~N}$ & $113 \mathrm{~N}$ & $112 \mathrm{~N}$ & $117 \mathrm{~N}$ \\
5 & $102 \mathrm{~N}$ & $108 \mathrm{~N}$ & $107 \mathrm{~N}$ & $119 \mathrm{~N}$ \\
6 & $122 \mathrm{~N}$ & $126 \mathrm{~N}$ & $125 \mathrm{~N}$ & $131 \mathrm{~N}$ \\
7 & $117 \mathrm{~N}$ & $119 \mathrm{~N}$ & $124 \mathrm{~N}$ & $128 \mathrm{~N}$ \\
8 & $110 \mathrm{~N}$ & $117 \mathrm{~N}$ & $109 \mathrm{~N}$ & $114 \mathrm{~N}$ \\
9 & $116 \mathrm{~N}$ & $121 \mathrm{~N}$ & $115 \mathrm{~N}$ & $119 \mathrm{~N}$ \\
10 & $117 \mathrm{~N}$ & $123 \mathrm{~N}$ & $119 \mathrm{~N}$ & $126 \mathrm{~N}$ \\
11 & $107 \mathrm{~N}$ & $118 \mathrm{~N}$ & $115 \mathrm{~N}$ & $121 \mathrm{~N}$ \\
12 & $115 \mathrm{~N}$ & $124 \mathrm{~N}$ & $117 \mathrm{~N}$ & $128 \mathrm{~N}$ \\
13 & $111 \mathrm{~N}$ & $115 \mathrm{~N}$ & $111 \mathrm{~N}$ & $124 \mathrm{~N}$ \\
14 & $130 \mathrm{~N}$ & $132 \mathrm{~N}$ & $128 \mathrm{~N}$ & $140 \mathrm{~N}$ \\
15 & $122 \mathrm{~N}$ & $128 \mathrm{~N}$ & $127 \mathrm{~N}$ & $132 \mathrm{~N}$ \\
16 & $120 \mathrm{~N}$ & $130 \mathrm{~N}$ & $129 \mathrm{~N}$ & $134 \mathrm{~N}$ \\
\hline
\end{tabular}

Individual force needed to enter the $4 \mathrm{~mm}$ shaver blade into the distal syndesmosis with the following fixation: 1SB, 2SB, 1 screw, 2 screws.

difference was found between the two-SB and one-screw methods $(\mathrm{p}=0.24)$.

\section{Comparison of the AITFL+IOL cut with the SB and screw methods}

For the one-SB method, $\mathrm{t}(15)=43.84 ;(\mathrm{p}<0.001 ; 95 \%$ CI 55.18 to 60.81$)$, the two-SB t $(15)=38.20(\mathrm{p}<0.001 ; 95 \%$ CI 59.66 to 66.71$)$, the one-screw revealed $\mathrm{t}(15)=43.23 \quad(\mathrm{p}<0.001$; $95 \%$ CI 58.11 to 64.13$)$ and the two-screw had a t $(15)=39.60$ ( $\mathrm{p}<0.001 ; 95 \%$ CI 63.98 to 71.26 ), respectively (table 3 ).

\section{DISCUSSION}

The results of this study showed a significant decrease in the force required with any of the ligaments transected. The most notable difference was shown when the AITFL, PITFL and the IOL were all transected, as expected since this would reflect the greatest instability. Additionally, the type of fixation also impacted joint stability, evidenced by the force required. The greatest amount of force needed was seen with the two-screw fixation, suggesting that this method provides the greatest stability for the syndesmosis. These results were consistent in the average forces required, as well as intraspecimen measurements.

Stable isolated syndesmotic lesions can be treated conservatively, while unstable lesions require surgery. ${ }^{9}$ The indication for surgery includes a positive squeeze test with a positive external rotation test, tenderness over the anterior interosseous ligament, $5 \mathrm{~cm}$ proximal to the ankle joint and injury to the deltoid ligament or the posterior inferior tibiofibular ligament on MRI. ${ }^{211-13}$

The radiographic measurements of an intact syndesmosis have great variability. ${ }^{14}$ The 'tibiofibular clear space' and the 'tibiofibular overlap' are the most frequently used radiographic measurements to determine instability. ${ }^{15} 16$ Although MRI can accurately identify individual ligamentous ruptures, studies that use the clear space and overlap measurements, unfortunately, do not correlate with syndesmotic instability. Also, since an MRI is not a dynamic test, it cannot diagnose abnormal joint movement. ${ }^{313}$ 

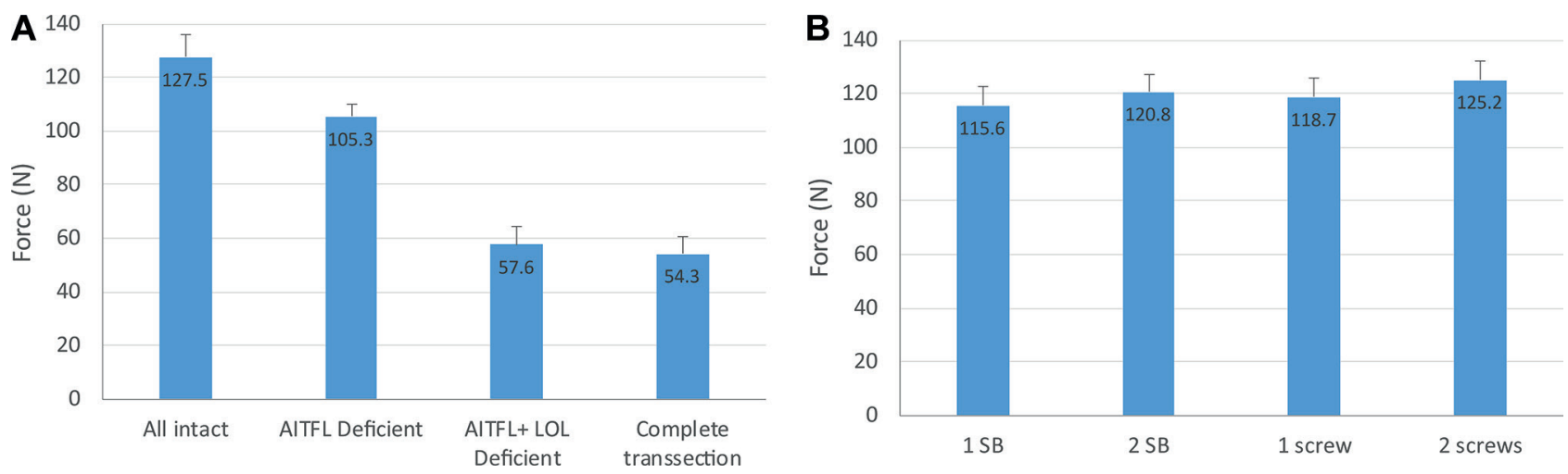

Figure 4 Mean Force: Measured Mean force needed based on the ligament cut methods and for the application of the SB and screw methods. AITFL, anterior inferior tibiofibular ligament; IOL, interosseous ligament.

In grade II syndesmotic lesions, it is unclear which injuries should be treated conservatively or surgically. Currently, we are unable to quantify syndesmotic (in)stability arthroscopically, since there are no established arthroscopic criteria available. ${ }^{17}$ There is a consensus among experts that arthroscopy is the gold standard in the evaluation of syndesmotic instability. ${ }^{918}$ During arthroscopy, isolated syndesmotic instability can be assessed by inserting a $4 \mathrm{~mm}$ shaver tip into the anterior distal syndesmosis of the ankle to determine if there is syndesmotic disruption. This allows for the evaluation of distal syndesmotic joint space opening, while moving the ankle in external rotation. More than $4 \mathrm{~mm}$ of joint space opening has been accepted as being indicative of instability. ${ }^{9}$

Ankle arthroscopy is a more sensitive method in the detection of syndesmotic instability compared with stress radiography. ${ }^{1} 121719$ Although there is still no consensus on how much diastasis the syndesmotic joint complex allows for to maintain physiological stability in the anterior compared with the posterior part of the syndesmosis, there is a known variation in distance between the tibia and fibula over the joint line. ${ }^{19}$ Especially the central part-that contains the tibiofibular syndesmotic recess-has variable differences in its dimensions. ${ }^{20}$ Another topic of debate remains the location and the required force application to arthroscopically measure syndesmotic diastasis. ${ }^{9}$ Most authors agree to confirm arthroscopic stabilisation of the distal tibiofibular joint in cases of doubt to avoid progression to chronic syndesmotic instability. ${ }^{17}$ In our cadaveric study, the force required to enter the distal syndesmosis was tested as recommended arthroscopically.

A study by Takao et al demonstrated the value of arthroscopy as an accurate indicator for a tibiofibular syndesmotic tear. ${ }^{17}$ In cases of subtle syndesmotic instability, however, every patient would require arthroscopic surgery as an invasive diagnostic tool. It can also be challenging to identify subtle syndesmotic instability in less experienced arthroscopic surgeons. Furthermore, an arthroscopic finding of a ruptured anterior syndesmotic

Table 3 Fixation w/AITFL+ IOL deficiency t-test

\begin{tabular}{llll}
\hline & $\mathbf{t}(\mathbf{1 5 )}$ & $95 \% \mathrm{Cl}$ & P value \\
\hline SB & 43.84 & 55.18 to 60.81 & $<0.001$ \\
2SB & 38.2 & 59.66 to 66.71 & $<0.001$ \\
1 screw & 43.23 & 58.11 to 64.13 & $<0.001$ \\
2 screws & 39.6 & 63.98 to 71.26 & $<0.001$
\end{tabular}

Significant differences found based on the fixation method with the AITFL+IOL cut. AITFL, anterior inferior tibiofibular ligament; IOL, interosseous ligament. ligament does not unequivocally mean there is syndesmotic instability, because the interosseous complex (ligament and membrane) cannot reliably be assessed during ankle arthroscopy.

Indicators of instability, such as fibular subluxation, deltoid ligament injuries and posterior malleolar fractures should also be taken into account in the preoperative planning. ${ }^{21}$

Previous studies present a variety of methods and cut-off points to differentiate stable from unstable syndesmotic injuries. Leeds indicated movement $\geq 2 \mathrm{~mm}$ between the tibia and fibula as a diagnosis of instability. ${ }^{22}$ Wagener et al confirmed to instability if at least $3 \mathrm{~mm}$ of the test probe could swiftly be inserted and twisted in the syndesmosis. ${ }^{19}$ Another syndesmotic evaluation method defines instability if the degree of fibular dislocation from the tibiofibular joint is more than $1 \mathrm{~mm} .^{12}$

Current literature does not provide us with clear and reproducible guidelines on the amount of displacement or degree of diastasis that are required to indicate syndesmotic stabilisation. ${ }^{1918}$ Also, most studies do not mention the testing location or necessary force used to detect syndesmotic instability. Van de Bekerom et al showed that a lateral force of $100 \mathrm{~N}$ to the ankle mortise seems appropriate to diagnose instability and that forces of $>100 \mathrm{~N}$ did not show a substantial increase in displacement. ${ }^{19}$

The main limitations of this study are that all measurements were taken on cadaveric specimens with a large age range without specific information on previous ankle injury. The sample size was based on the known incidence of syndesmotic injuries, as well as the intrareliability of the testing parameters. However, this study still has a relatively small sample size. Although the testing was performed by only one surgeon, the force to enter the distal syndesmosis can be operator-dependent. Additionally, having more than one observer would allow for us to assess the interreliability of observers and strengthen the reliability of this testing method. Also, several authors have concluded that the assessment of sagittal plane movement appears to be a more sensitive test of inferior tibiofibular instability than assessment in the coronal plane. This study only looked at coronal plane syndesmotic instability. Furthermore, most of the aforementioned studies that assess distal syndesmotic instability are related to injuries with combined ankle fractures involved. Caution must be taken in interpreting the results of these studies related to ankle syndesmotic injuries without a fracture.

\section{Clinical relevance}

This cadaveric study presents the next step towards the validation of arthroscopic testing of syndesmotic injuries. Currently, there 
are cadaveric studies published that are not directly applicable to intraoperative utilisation. Particularly, in the case of grade II injuries, identifying the stable versus unstable ankle remains a challenge, since both radiographic imaging tools and clinical diagnostic tests are inconclusive. Even arthroscopic evaluation has relied heavily on surgeon experience and expert opinion without a standardised, validated measurement tool. The data from this study need to be clinically correlated to ultimately assist in improving the outcome of patients with syndesmotic ankle injuries. Therefore, our study offers to bridge the gap to the development of arthroscopic tools that can identify the need for surgical fixation to the syndesmosis based on the laxity of specific ankle ligaments that contribute to subtle instability. Furthermore, the methods used in this study are reliable and accurate, allowing surgeons to examine the syndesmosis during arthroscopic surgery.

\section{CONCLUSION}

Together with stress radiographs and MRI, there are helpful clinical tests available to indicate syndesmotic ligament injury. Nonetheless, there is no best-evidence criteria to evaluate instability. This cadaveric study provides biomechanical data that can assist surgeons in the arthroscopic evaluation of syndesmotic injuries.

Acknowledgements The authors thank Dr. Philippe Landreau, Fawaz Hamzie and Miss Khloud Sebak from the Aspetar Surgical Training Center for their availability to perform the first cadaveric pilot case that initiated this study and allowed for preparation of the methodology.

Contributors All included authors have contributed to the study design, implementation, writing and/or editing of this manuscript. PD'H: Study conception and design, data acquisition analysis and interpretation; manuscript writing, editing and submission. MCC: Data analysis and interpretation; manuscript writing, editing and submission. MVH: Study conception and design, manuscript editing and submission. VM: Study design, data interpretation, manuscript editing. KA: Study conception and design, data acquisition, analysis and interpretation. TM: Study design, data acquisition, analysis and interpretation. FHF: Data interpretation, manuscript review. J-FK: Study concept, data interpretation, manuscript review.

Funding The authors have not declared a specific grant for this research from any funding agency in the public, commercial or not-for-profit sectors.

Competing interests None declared.

Patient consent for publication Not required.

Ethics approval This study was approved by the institutional review board for the use of cadaveric specimens.

Provenance and peer review Not commissioned; externally peer reviewed.

Open access This is an open access article distributed in accordance with the Creative Commons Attribution Non Commercial (CC BY-NC 4.0) license, which permits others to distribute, remix, adapt, build upon this work non-commercially, and license their derivative works on different terms, provided the original work is properly cited, an indication of whether changes were made, and the use is noncommercial. See: http://creativecommons.org/licenses/by-nc/4.0/.

\section{REFERENCES}

1 van Dijk CN, Longo UG, Loppini M, et al. Classification and diagnosis of acute isolated syndesmotic injuries: ESSKA-AFAS consensus and guidelines. Knee Surg Sports Traumatol Arthrosc 2016;24:1200-16.

2 Ebraheim NA, Taser F, Shafiq Q, et al. Anatomical evaluation and clinical importance of the tibiofibular syndesmosis ligaments. Surg Radiol Anat 2006;28:142-9.

3 Beumer A, Valstar ER, Garling EH, et al. External rotation stress imaging in syndesmotic injuries of the ankle: comparison of lateral radiography and radiostereometry in a cadaveric model. Acta Orthop Scand 2003;74:201-5

4 van den Bekerom MP, Haverkamp D, Kerkhoffs GM, et al. Syndesmotic stabilization in pronation external rotation ankle fractures. Clin Orthop Relat Res 2010;468:991-5.

5 van den Bekerom MP, Mutsaerts EL, van Dijk CN. Evaluation of the integrity of the deltoid ligament in supination external rotation ankle fractures: a systematic review of the literature. Arch Orthop Trauma Surg 2009;129:227-35.

6 Woods C, Hawkins R, Hulse M, et al. The football association medical research programme: an audit of injuries in professional football: an analysis of ankle sprains. Br J Sports Med 2003:37:233-8.

7 Kofotolis ND, Kellis E, Vlachopoulos SP. Ankle sprain injuries and risk factors in amateur soccer players during a 2-year period. Am J Sports Med 2007;35:458-66.

8 Roemer FW, Jomaah N, Niu J, et al. Ligamentous injuries and the risk of associated tissue damage in acute ankle sprains in athletes: a cross-sectional MRI study. Am J Sports Med 2014;42:1549-57.

9 van Dijk CN, Longo UG, Loppini M, et al. Conservative and surgical management of acute isolated syndesmotic injuries: ESSKA-AFAS consensus and guidelines. Knee Surg Sports Traumatol Arthrosc 2016;24:1217-27.

10 Cohen J. Statistical power analysis for the behavioral sciences. 2nd edn. Hillsdale, NJ: Lawrence Erlbaum Associates, 1988.

11 Sman AD, Hiller CE, Refshauge KM. Diagnostic accuracy of clinical tests for diagnosis of ankle syndesmosis injury: a systematic review. Br J Sports Med 2013;47:620-8

12 Vopat ML, Vopat BG, Lubberts B, et al. Current trends in the diagnosis and management of syndesmotic injury. Curr Rev Musculoskelet Med 2017;10:94-103.

13 Oae K, Takao M, Naito K, et al. Injury of the tibiofibular syndesmosis: value of MR imaging for diagnosis. Radiology 2003;227:155-61.

14 Xenos JS, Hopkinson WJ, Mulligan ME, et al. The tibiofibular syndesmosis. Evaluation of the ligamentous structures, methods of fixation, and radiographic assessment. J Bone Joint Surg Am 1995;77:847-56.

15 Harper MC. An anatomic and radiographic investigation of the tibiofibular clear space. Foot Ankle 1993;14:455-8.

16 Pneumaticos SG, Noble PC, Chatziioannou SN, et al. The effects of rotation on radiographic evaluation of the tibiofibular syndesmosis. Foot Ankle Int 2002;23:107-11.

17 Takao M, Ochi M, Oae K, et al. Diagnosis of a tear of the tibiofibular syndesmosis. The role of arthroscopy of the ankle. J Bone Joint Surg Br 2003:85:324-9.

18 Calder JD, Bamford R, Petrie A, et al. Stable versus unstable grade II high ankle sprains: a prospective study predicting the need for surgical stabilization and time to return to sports. Arthroscopy 2016;32:634-42.

19 van den Bekerom MP. Diagnosing syndesmotic instability in ankle fractures. World $\mathrm{J}$ Orthop 2011:2:51-6.

20 Ebraheim NA, Lu J, Yang H, et al. Radiographic and CT evaluation of tibiofibular syndesmotic diastasis: a cadaver study. Foot Ankle Int 1997:18:693-8.

21 Hunt KJ, Phisitkul P, Pirolo J, et al. High ankle sprains and syndesmotic injuries in athletes. J Am Acad Orthop Surg 2015;23:661-73.

22 Leeds HC, Ehrlich MG. Instability of the distal tibiofibular syndesmosis after bimalleolar and trimalleolar ankle fractures. J Bone Joint Surg Am 1984;66:490-503. 